Canad. Math. Bull. Vol. 21 (1), 1978

\title{
THE SPECTRAL RADIUS OF A NON-NEGATIVE MATRIX
}

\author{
BY \\ A. BERMAN \\ Union Carhide Corporation, Nuclear Division* \\ Computer Sciences Division \\ Oak Ridge, Tennessee \\ June, 1976
}

\begin{abstract}
A max min formula for the spectral radius of a non-negative matrix is derived from a characterization of non-singular $M$-matrices in terms of diagonal stability.
\end{abstract}

The matrices in this note are real and square. A matrix $A$ is said to be in a class $Z$ if it is of the form $A=\lambda I-B$, where $B$ is non-negative $\left(B_{i i} \geq 0\right)$. A matrix $A$ in $Z$ is called a non-singular $M$-matrix if $\lambda>\rho(B)$, the spectral radius of $B$. A matrix $A$ is said to be diagonally stable if there exists a positive diagonal matrix $D\left(D_{i i}>0\right)$ such that $A D+D A^{t}$ is positive definite. Diagonally stable matrices arise in the study of predator-prey systems, e.g. Krikorian [3], and of composite dynamical systems, e.g. Araki [1]. An excellent survey of the theory and many applications of $M$-matrices is given in Plemmons [4].

In this note we obtain an expression for the spectral radius, $\rho(B)$, of a non-negative matrix $B$. The results follow from two characterizations of diagonally stable matrices. The first is general. The second is restricted to the class $Z$.

Lemma 1. (Barker, Berman and Plemmons [2]) $A$ is diagonally stable if and only if for every non-zero positive semidefinite matrix $S$, there exists an index $i$ such that $(S A)_{i i}$ is positive.

Lemma 2. (Tartar [5], Araki [1] If A\&Z, then diagonal stability is a necessary and sufficient condition for $A$ to be a non-singular M-matrix.

Combining the two results yields the desired expression for $\rho(B)$.

THEOREM. Let $B$ be a non-negative matrix.

\section{Then}

$$
\rho(B)=\max \min \frac{(S B) i i}{S_{i i}}
$$

\footnotetext{
*Prime contractor for the U.S. Energy Research and Development Administration Received by the editors November 8, 1976 and revised May 13, 1977.

Key Words and Phrases: Spectral radius, non-negative matrices, positive semidefinite matrices $M$-matrices, diagonal stability.

Computing Review Categories: 5.11, 5.15.

American Mathematical Society Subject Classification: 15A15, 15A48.
} 
where the minimum is computed over all indices $i$ such that $S_{i i}>0$ and the maximum is taken over all non-zero positive semidefinite matrices $S$.

Proof. For every $\varepsilon>0,(\rho(B)+\varepsilon) I-B$ is a non-singular $M$-matrix. Thus $\rho(B) \geq \max \min \left(S B_{i i} / S_{i i}\right)$. On the other hand $\rho(B) \leq \max \min \left(S B_{i i} / S_{i i}\right)$ since $\rho(B) I-B$ is not a non-singular $M$-matrix.

Notice that choosing $S=\left(S_{i j}\right)=(1)$, yields the well known lower bound

$$
\rho(B) \geq \min _{j} \sum_{i} b_{i j}
$$

\section{REFERENCES}

1. M. Araki, Applications of M-matrices to the Stability Problems of Composite Dynamical Systems. J. Math. Anal. and Appl., 52 (1975), 309-321.

2. G. P. Barker, A. Berman and R. J. Plemmons, Positive Diagonal Solutions to the Lyapunov Equations, University of Wisconsin, M. R. C. Report No. 1713. To appear in Linear and Multilinear Algebra.

3. N. Krikorian, Private communication.

4. R. J. Plemmons, A survey of M-matrix Characterizations I: Non-singular M-matrices, University of Wisconsin. M. R. C. Report No. 1651. To appear in Linear Alg. and Appl.

5. L. Tartar, Une Nouvelle Caracterisation des M-matrices, R. I. R. O., No. R-3 (1971), $127-128$.

Department of Mathematics, ISRAEL INSTITUTE OF TECHNOLOGY

TECHNION, HAIFA 